\title{
INFLUENCIA DE LA TRANSPARENCIA EN LA GESTIÓN ORGANIZACIONAL DEL GOBIERNO REGIONAL DE TACNA, PERIODO $2011-2014$
}

\author{
INFLUENCE OF TRANSPARENCY ON THE ORGANIZATIONAL MANAGEMENT OF THE REGIONAL \\ GOVERNMENT OF TACNA, PERIOD 2011 - 2014
}

Pedro Casiano Rodas Alejos ${ }^{1}$

Presentado: 07/04/2018

Aceptado: 07/05/2018

Publicado online:27/12/2018

\section{RESUMEN}

El presente trabajo de investigación tiene como objetivo principal el determinar la influencia de la transparencia en la gestión organizacional del Gobierno Regional de Tacna durante el período 2011 - 2014; se consideró una muestra de 266 personas; el tipo de investigación fue básico de nivel correlacional, siendo el diseño no experimental; se encontró que existe influencia significativa de la percepción sobre la transparencia en la gestión organizacional que caracteriza a la entidad. Se encontró que el nivel de trasparencia que percibe la población electoral de Tacna sobre su Gobierno Regional es regular, y que la dimensión "Investigación y sanción" fue la más destacada, y el focalizado a mejorar la "Institucionalidad y eficiencia"; el nivel de gestión organizacional que se percibe es inadecuado, siendo la dimensión más destacada el "Desarrollo organizacional", y el focalizado a mejorar la "Gobernabilidad democrática".

Palabras claves: Transparencia, gestión organizacional, desarrollo

\section{ABSTRACT}

The present work of investigation has as principal aim determine the influence of the transparency in the management organizacional of the Regional Government of Tacna during the period 2011 - 2014; it was considered to be a sample of 266 persons; the type of investigation was basic of level correlacional, being the not experimental design; one thought

\footnotetext{
${ }^{1}$ Magíster en Gestión y Políticas Públicas
} 
that significant influence of the perception exists on the transparency in the management organizational that it characterizes to the entity. One found that the level of trasparencia that it perceives the electoral population of Tacna on his Regional Government is regular, and that the dimension "Investigation and sanction" was the most out-standing, and the focused one to improving the "Institucionality and efficiency"; the level of management organizational that is perceived is inadequate, being the most out-standing dimension the "Development organizational", and the focused one to improving the "Democratic Gobernability".

Key words: Transparency, management organizational.

\section{INTRODUCCIÓN}

Una actitud transparente por parte de las instituciones del Estado, requiere de una alta predisposición de los directivos y funcionarios de mostrar no solamente los aspectos institucionales, financieros, administrativos, otros que la normatividad respectiva obliga a enseñar a cualquier poblador, sino de desarrollar un comportamiento ético y predicar con el ejemplo, respecto a usar de forma adecuada los recursos públicos. Por tanto, es prioritario que los funcionarios públicos estén dispuestos a comportarse de manera transparente, para lo cual es importante fortalecer el sistema de incentivos que permita fomentar tal comportamiento. En el Perú, a través de los medios de comunicación, los pobladores se han enterado de casos probados de mal uso de los recursos públicos, asociados a temas de corrupción, y ello afecta de forma significativa en la credibilidad de la población en sus autoridades; de ahí la relevancia del presente trabajo de investigación, que busca proponer algunas recomendaciones conducentes a mejorar los niveles de transparencia en las entidades públicas. El trabajo de investigación tiene como objetivo principal el determinar la influencia de la transparencia en la gestión organizacional del Gobierno Regional de Tacna. Es prioritario precisar que las organizaciones del Estado, deben tender a ser transparentes en sus decisiones, buscando dar facilidades de acceso a la ciudadanía sobre información que le permita entender la forma de cómo se están ejecutando los principales proyectos de inversión que impulsan la mejora de los indicadores socio-económicos.

En el Perú, se han hecho público diversos casos relacionado con la corrupción y el uso indebido de los recursos públicos por parte de los directivos y funcionarios públicos, lo cual afecta de forma significativa en la credibilidad que se tiene en el accionar de nuestras autoridades. Por tanto, las organizaciones del Estado, deben tender a ser transparentes en sus decisiones, y facilitar el acceso al poblador de diversa información que le permita entender la forma de cómo se están ejecutando los principales proyectos de inversión pública que impulsan la mejora de los indicadores sociales, económicos, culturales, otros de su jurisdicción. Por tanto, dicha problemática se aborda en la presente investigación, a través de la percepción ciudadana sobre el nivel de transparencia institucional que caracteriza al Gobierno Regional de Tacna, y ello como logro incidir en su gestión.

Gonzáles (2008) desarrolló una investigación denominada "Gestión Municipal para la superación de la pobreza donde concluyó que una intervención sostenible debe apuntar a la 
mejora de la demanda y oferta laboral donde el municipio de forma efectiva interviene en la demanda de empleo. Abdó (2009) refuerza que el medio más eficaz para terminar con la corrupción y la cultura del silencio es la transparencia de la actividad gubernamental y el acceso a la información, puesto que permite fortalecer la democracia y consolida el estado de derecho. Munch (2009) señala sobre la transparencia institucional que se trata del "Compromiso que establece una organización gubernamental por dar a conocer al público que lo solicite la información existente sobre un asunto público..." y es el "conjunto de actividades, estructuras, procedimientos, reglas, normas e incentivos establecidos dentro del servicio público que proporciona, monitorea y controla directa o indirectamente los productos y servicios del sector público". (Ingraham, 2005).

El 13 de julio del 2002, en la gestión del Presidente Alejandro Toledo Manrique, se promulgó la Ley no 27806 denominada "Ley de transparencia y acceso a la información pública", donde la política de transparencia institucional tiene como objetivo principal el de dotar a las instituciones públicas, privadas y a la ciudadanía, de los respectivos instrumentos orientados a la prevención, investigación y sanción de actos de corrupción. Rowland (2008) define la corrupción como "el fenómeno por medio del cual, un funcionario público es impulsado a actuar de modo distinto a los estándares normativos del sistema para favorecer intereses particulares a cambio de una recompensa....)". Escalante (2009) establece tres tipos de causas que posibilitan la aparición de la corrupción: causas formales, causas culturales y causas materiales. Schepherd (2002) señala que existe un consenso sobre la urgencia de que la administración pública cambie, con la finalidad de priorizar un proceso de crecimiento económico, pero con inclusión social. Diez y Redondo (2007) plantean que "El éxito de una cultura de gestión pública se está convirtiendo en el problema central del desarrollo de los países avanzados".

\section{METODOLOGÍA}

El tipo de investigación es básico, nivel explicativo y correlacional. Diseño no experimental. La población de estudio está constituida por los electores del Gobierno Regional de Tacna (GRT), para el período 2011 - 2014; que fueron aproximadamente 160000 personas (51\% mujeres y $49 \%$ hombres). La muestra fue de 266 electores que fueron encuestados de forma aleatoria. Los instrumentos que se aplicaron fueron dos: Cuestionario para determinar la percepción de la población sobre la transparencia institucional que caracteriza al Gobierno Regional de Tacna y cuestionario para determinar la percepción de la población sobre la gestión organizacional que caracteriza al Gobierno Regional de Tacna. Para el análisis general de las variables, se elaboró una escala de valoración en base a la Escala de Likert (entre 1 = muy en desacuerdo con lo afirmado en el ítem y $5=$ muy de acuerdo con lo afirmado en el ítem). En lo que respecta a la escala de valoración para la variable "Transparencia", se considera los valores extremos que oscilan entre 17 y 85 puntos; y para la variable "Gestión organizacional", se tienen los valores extremos que oscilan entre 20 y 100 puntos.

\section{RESULTADOS}

La población, que considera que en el GRT existe un área que logra informar y poner a disposición de la ciudadanía la información generada por la entidad; y opina que los aspectos a mejorar son el efectuar periódicamente rendiciones de cuentas sobre la ejecución del plan de desarrollo regional y del presupuesto institucional por toda fuente de financiamiento; y el proceso de transparencia y acceso en los niveles de contratación, compras y servicios del 
Estado. Se debe caracterizar la contratación de su personal en base a la meritocracia, y después aplicar evaluaciones de desempeño por objetivos; y se debería impulsar la participación de la ciudadanía en instancias afines con la formulación y/o ejecución de planes y programas. LA población percibe una mejora en el mecanismo de control y seguimiento a las denuncias efectuadas por la ciudadanía pero que debería existir un mecanismo de control y seguimiento a las faltas cometidas por funcionarios en el ejercicio de la función pública, así mismo para contratistas y proveedores.

Según la variable "Transparencia", el valor medio general que fue de 45,36 y una desviación típica de 12,224, que refleja un nivel medio o regular (Fig. 01). Al comparar por dimensión, se encontró que el más destacado fue el denominado "Investigación y sanción", y el focalizado a mejorar "Institucionalidad y eficiencia". Al comparar por jurisdicción, los valores medios más altos se dieron en el distrito de Coronel Gregorio Albarracín Lanchipa. (fig.02).

Figura 01: Transparencia (por distrito)

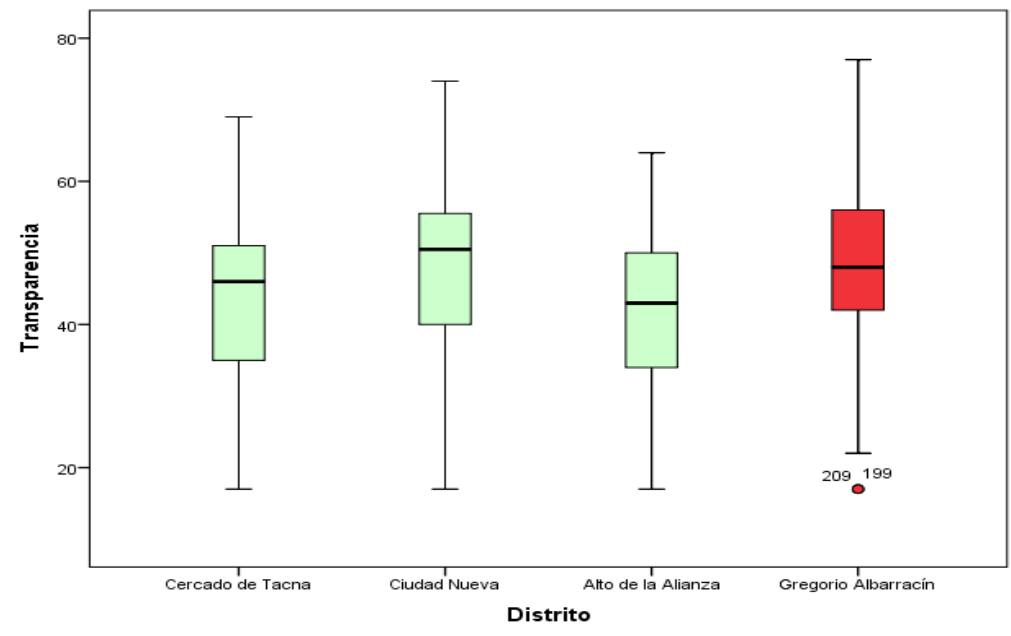

Figura 02: Media por distrito 


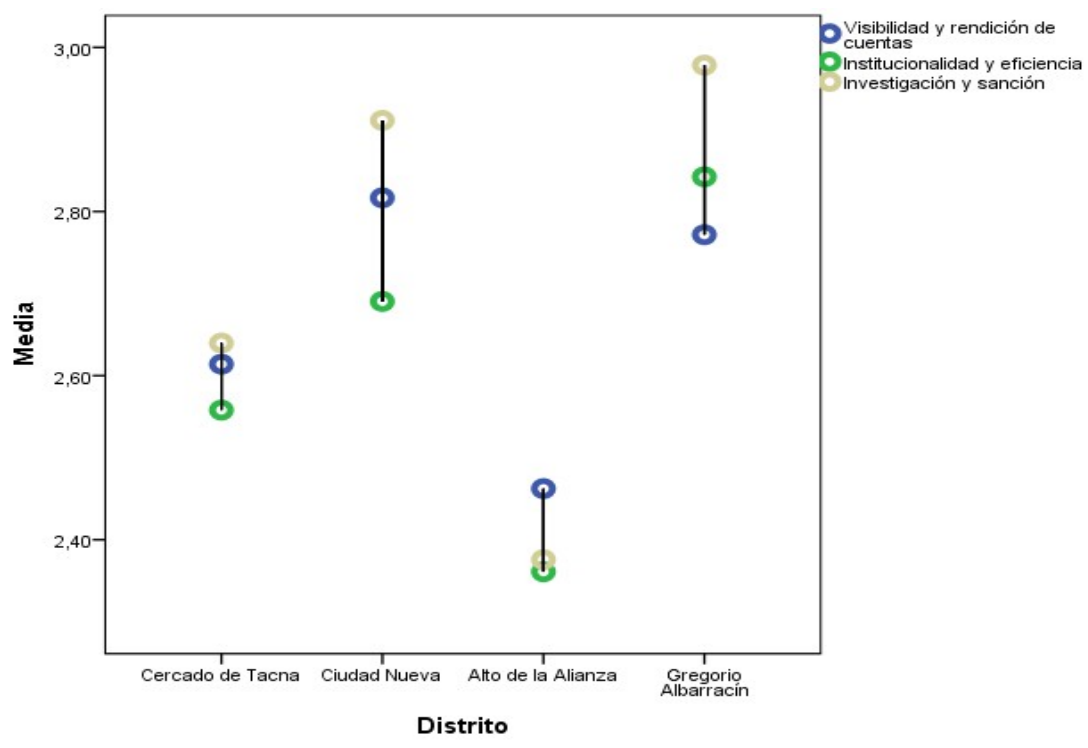

Según Desarrollo Organizacional, se puede inferir que la población electoral de la región Tacna percibe que el nivel de desenvolvimiento organizacional que caracterizó al Gobierno Regional durante el periodo 2011 - 2014 fue inadecuado (Fig. 03) así como el de finanzas públicas (Fig. 04), servicios y proyectos (Fig. 05), gobernabilidad democrática (Fig. 06) y gestión organizacional (Fig.07)

Figura 03: Desarrollo organizacional

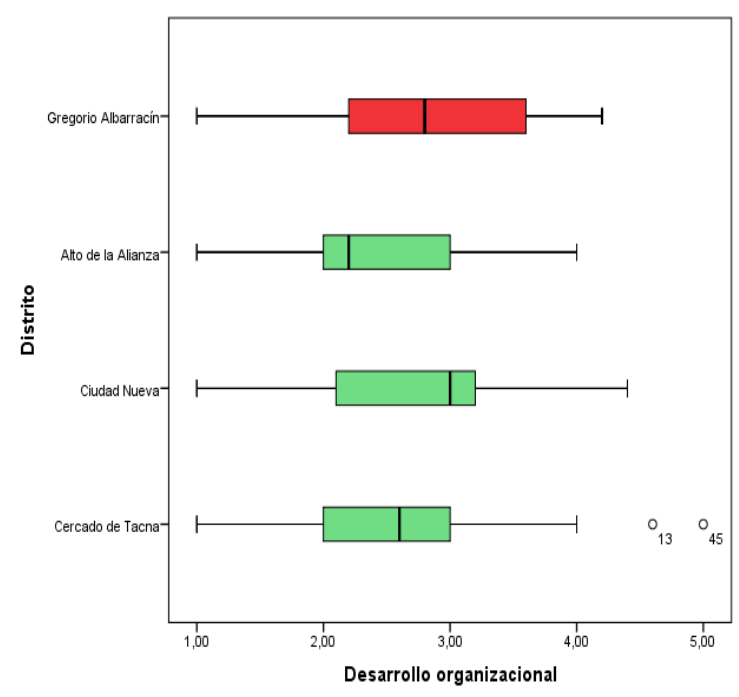

Figura 05: Servicios y proyectos
Figura 04: Finanzas públicas

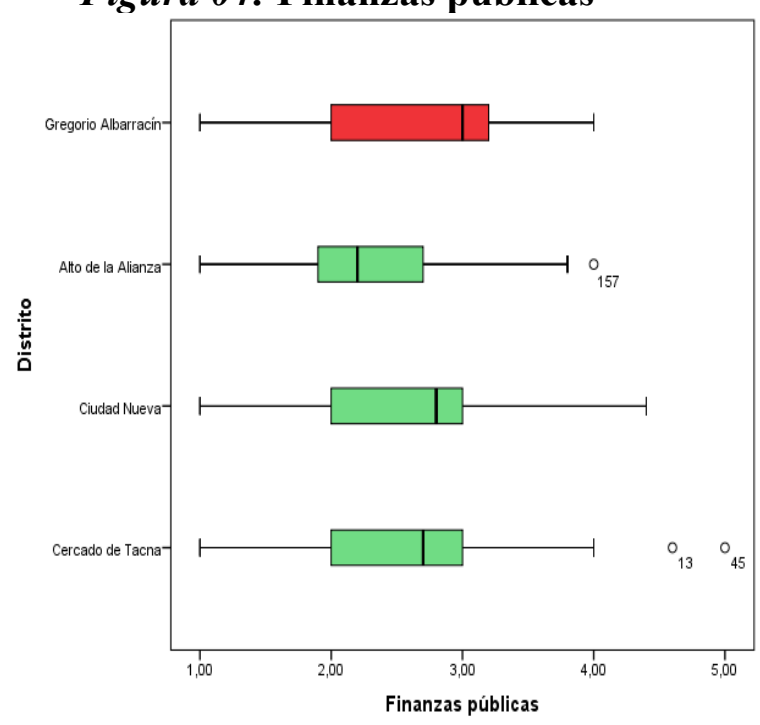



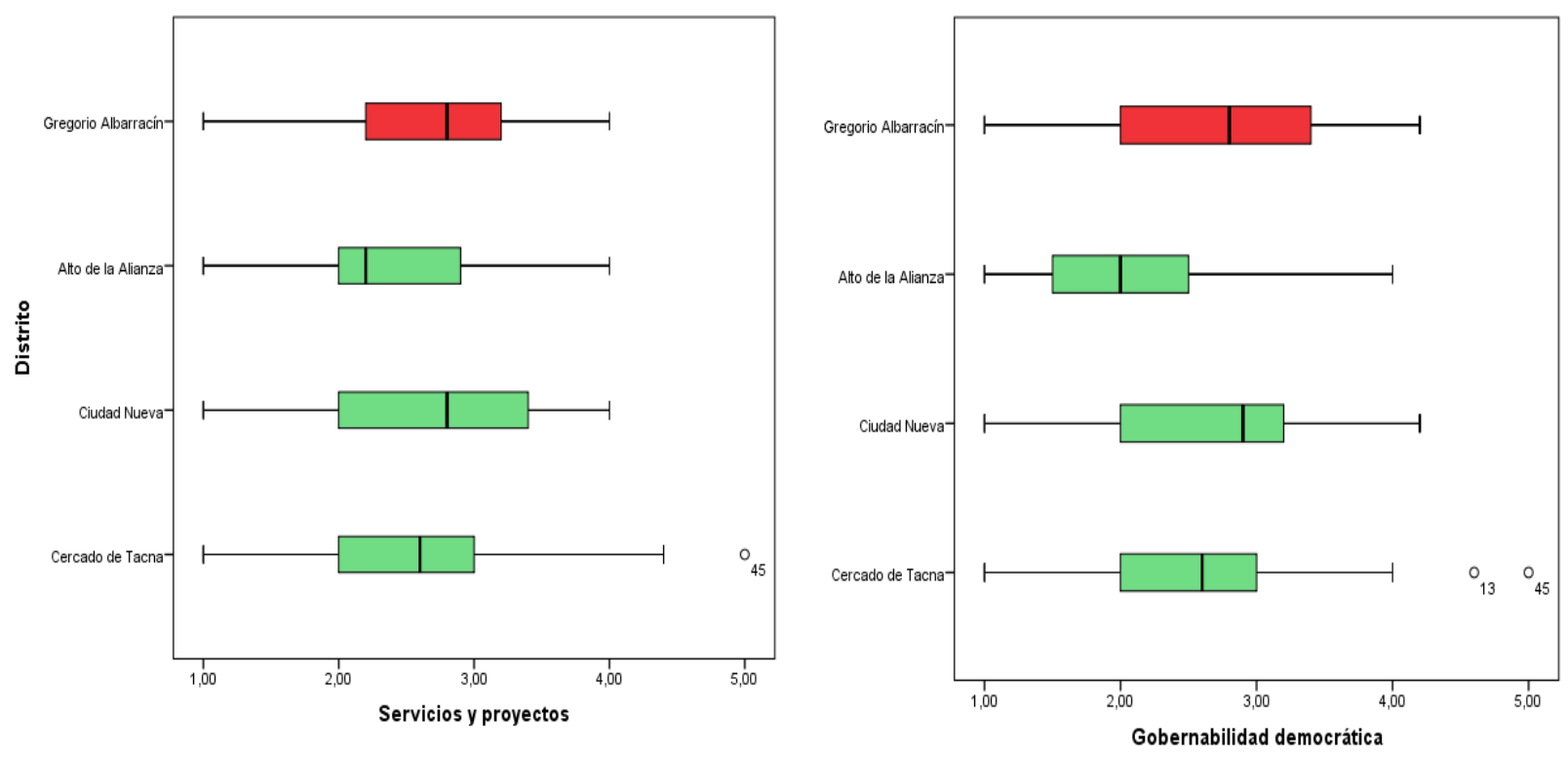
Figura 06: Gobernabilidad
democrática

Figura 07: Gestión organizacional

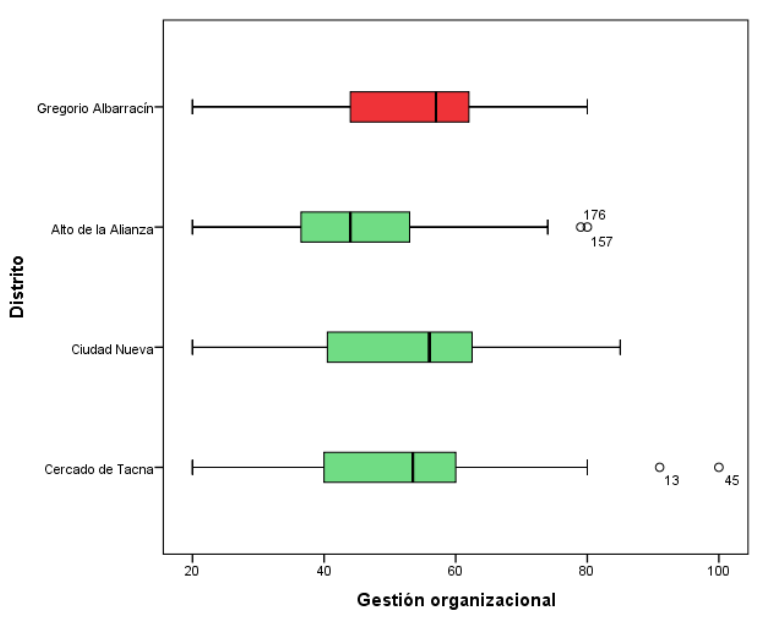

DISCUSIÓN

El trabajo de investigación se centró en una muestra de 266 electores del actual Gobierno Regional de Tacna, para el período 2011 - 2014. Se concluye que existe influencia significativa de la percepción sobre la transparencia en la gestión organizacional que caracteriza a la entidad pública analizada $(p=0,000)$. Existe influencia significativa $(p=0,000)$ de la visibilidad y rendición de cuentas sobre la gestión organizacional del Gobierno Regional de Tacna; también que el nivel de trasparencia que percibe la población electoral de Tacna sobre su Gobierno Regional es regular. Además, el nivel de gestión organizacional que se percibe es inadecuado. 
Al comparar por dimensión, se encontró que el más destacado fue el denominado "Desarrollo organizacional", y el focalizado a mejorar "Gobernabilidad democrática". Con la finalidad de mejorar el nivel de transparencia que percibe la población sobre la gestión del Gobierno Regional de Tacna, se recomienda a la Alta Dirección de dicha entidad el impulsar e implementar un Programa Regional de Transparencia, concebido como una herramienta y expresión metodológica de los contenidos y acciones, conceptualmente definidos por la Política Nacional de Integridad y lucha contra la Corrupción, cuya aplicación en la gestión pública promoverá la prevención de la corrupción; el cual debería centrarse en cuatro componentes: Acceso a la información: Que implica que la gestión debería priorizar el derecho fundamental de las personas a conocer el manejo de la gestión pública e instrumento de participación ciudadana que genera inclusión social, permite a los ciudadanos conocer el destino y uso de los recursos públicos. Ética pública: Debería abarcar la promoción de una cultura ética basada en principios, valores y conductas que permitan el desarrollo de la gestión pública más plena y armónica posible. Rendición de cuentas: De forma periódica, la gestión regional debería poner a consideración los resultados obtenidos en la gestión, así como el cumplimiento de compromisos asumidos con los actores sociales involucrados y sociedad civil en general y Control Social: Se deben implementar mecanismos o medios de seguimiento y participación activa de la sociedad organizada en los procesos acciones y resultados que desarrollan las instituciones regionales del Estado para el logro de sus objetivos.

Para mejorar la gestión organizacional en temas presupuestales, se recomienda a la Alta Dirección los siguientes aspectos: a. Fortalecer los mecanismos de asignación de recursos públicos para la provisión de bienes y servicios, b. Ampliar la cobertura de los programas presupuestales al $100 \%$ del presupuesto y c. Mejorar las capacidades sectoriales para la gestión de programas presupuestales. En temas de planificación, se recomienda fortalecer el sistema regional de planificación estratégica, a través de la creación del Centro de Planeamiento Regional - CEPLAR, que permita alinear el plan regional de desarrollo concertado con los planes locales de desarrollo, consolidar los instrumentos de seguimiento y evaluación de resultados, y priorizar la formulación, viabilización y ejecución de los proyecto de inversión emblemáticos en la región, afines como los recursos hídricos, de articulación regional, de industria y comercio, otros.

Para mejorar la gestión organizacional en temas de mejora a la atención al ciudadano, se recomienda a la Alta Dirección fortalecer la capacidad de generación de información de desempeño y su utilización para la toma de decisiones, promover progresivamente el acceso público a la información y la rendición de cuentas con un enfoque de Derechos Humano y fortalecer las capacidades del servidor público que permita mejorar el servicio que se le brinda a la ciudadanía.

\section{REFERENCIAS BIBLIOGRÁFICAS}

Abdó, J. (2009). "Transparencia y acceso a la información gubernamental". http://biblio.juridicas.unam.mx/libros/4/1646/17.pdf

Díez, J. y Redondo, C. (2007). "Administración de empresas". Ediciones Pirámide, S.A., Madrid (España). $621 \mathrm{pp}$.

Escalante, F. (2009). "La corrupción política: apuntes para un modelo teórico". En la revista Foro Internacional. Vol. XXX. № 2, México. Págs. 328-245. 
Gibson, J.; Ivancevich, J. y Donnelly, J. (2007). "Las organizaciones". Editorial Addison-Wesley Iberoamericana. (EEUU).

Gonzáles, D. (2008). "Gestión Municipal para la superación de la pobreza: Estrategias e instrumentos de intervención en el ámbito del empleo, a partir de la experiencia chilena". http://www.eclac.org/publicaciones/xml/0/12540/lcl1802e.pdf

Ingraham, P. (2005). "Administración de calidad total en las organizaciones públicas: Perspectivas y dilemas" en ¿De Burócratas a Gerentes? Las ciencias de la gestión aplicadas a la administración del Estado, p. 343.

Munch, L. (2009). "Ética y valores". Editorial Trillas. México, D.F.

Ponce, S. (2013). "Inversión pública y desarrollo económico regional". http://tesis.pucp.edu.pe/ repositorio/bitstream/handle/123456789/4837/ PONCE_SONO_STEFAHNIE_SOFIA_INVERSION.pdf?sequence=1

Rowland, M. "Visión contemporánea de la corrupción". http://www.iidh.ed.cr/comunidades/ redelectoral/docs/red_publinea/Cuaderno\%2049/III-M.Rowland-Vision.pdf

Schepherd, G. (2002). "Administración Pública en América Latina y El Caribe: En busca de un paradigma de reforma". En Administración Pública: El estado actual de la disciplina, p. 69. 